Douglas Mota Dias

\title{
Programação Genética Linear com Inspiração \\ Quântica
}

\section{Tese de Doutorado}

Tese apresentada como requisito parcial para obtenção do título de Doutor pelo Programa de Pós-Graduação em Engenharia Elétrica da PUC-Rio.

Orientador: Prof. Marco Aurélio Cavalcanti Pacheco 


\title{
Douglas Mota Dias
}

\section{Programação Genética Linear com Inspiração \\ Quântica}

Tese apresentada como requisito parcial para obtenção do grau de Doutor pelo Programa de Pós-Graduação em Engenharia Elétrica do Departamento de Engenharia Elétrica do Centro Técnico Científico da PUC-Rio. Aprovada pela comissão examinadora abaixo assinada.

\author{
Prof. Marco Aurélio Cavalcanti Pacheco \\ Orientador \\ Departamento de Engenharia Elétrica - PUC-Rio \\ Prof. André Vargas Abs da Cruz \\ Pesquisador do ICA/DEE/PUC-Rio
}

Prof. Antonio Carneiro de Mesquita Filho COPPE/UFRJ

Prof. Carlos Roberto Hall Barbosa

Departamento de Metrologia - PUC-Rio

Prof. Leandro dos Santos Coelho

PUC/PR

Prof. Omar Paranaíba Vilela Neto

Departamento da Ciência da Computação - UFMG

Prof. Renato Portugal

LNCC

Prof. José Eugênio Leal Coordenador do Centro Técnico Científico - PUC-Rio 
Todos os direitos reservados. Proibida a reprodução total ou parcial do trabalho sem autorização da universidade, do autor e do orientador.

\section{Douglas Mota Dias}

Graduou-se em Engenharia Elétrica (ênfase: Sistemas e Computação) pela Universidade Estadual do Rio de Janeiro (UERJ) em 2003. Mestre em Métodos de Apoio à Decisão pelo Departamento de Engenharia Elétrica da Pontifícia Universidade Católica do Rio de Janeiro (PUC-Rio), na linha de pesquisa de Inteligência Computacional, em 2005.

Ficha Catalográfica

Dias, Douglas Mota

Programação genética linear com inspiração quântica / Douglas Mota Dias ; orientador: Marco Aurélio Cavalcanti Pacheco. - 2010.

97 f. : il. ; $30 \mathrm{~cm}$

Tese (Doutorado) - Pontifícia Universidade Católica do Rio de Janeiro, Departamento de Engenharia Elétrica, 2010

Inclui bibliografia

1. Engenharia Elétrica - Teses. 2. Aprendizado de máquina. 3. Inteligência computacional. 4. Computação evolutiva. 5. Programação genética. 6. Algoritmos evolutivos com inspiração quântica. I. Pacheco, Marco Aurélio Cavalcanti. II. Pontifícia Universidade Católica do Rio de Janeiro. Departamento de Engenharia Elétrica. III. Título. 


\section{Agradecimentos}

Ao CNPq e à PUC-Rio pelos auxílios concedidos, sem os quais este trabalho não poderia ter sido realizado.

Ao meu orientador, Prof. Dr. Marco Aurélio Cavalcanti Pacheco, pelo estímulo e parceria na realização deste trabalho.

À Prof- ${ }^{\text {a }}$ Dr- ${ }^{\mathrm{a}}$ Marley M. B. R. Vellasco pela oportunidade, apoio e incentivo.

Aos professores que participaram da comissão examinadora.

A todos os professores e funcionários da PUC-Rio pelos ensinamentos e pelo auxílio.

Aos amigos e colegas da PUC-Rio e do laboratório ICA pela paciência durante as longas conversas sobre física quântica e computação evolutiva.

Aos meus familiares e amigos que de uma forma ou de outra me estimularam e ajudaram.

Aos meus pais, pelo apoio e incentivo.

À minha mãe, pela educação, dedicação e inabalável confiança.

À minha filha Maria Antônia, pela constante alegria e motivação.

À minha esposa Kátia, pelo carinho, apoio e compreensão incondicionais. 


\section{Resumo}

Dias, Douglas Mota; Pacheco, Marco Aurélio Cavalcanti. Programação Genética Linear com Inspiração Quântica. Rio de Janeiro, 2010. 97p. Tese de Doutorado - Departamento de Engenharia Elétrica, Pontifícia Universidade Católica do Rio de Janeiro.

A superioridade de desempenho dos algoritmos quânticos, em alguns problemas específicos, reside no uso direto de fenômenos da mecânica quântica para realizar operações com dados em computadores quânticos. Esta característica fez surgir uma nova abordagem, denominada Computação com Inspiração Quântica, cujo objetivo é criar algoritmos clássicos (executados em computadores clássicos) que tirem proveito de princípios da mecânica quântica para melhorar seu desempenho. Neste sentido, alguns algoritmos evolutivos com inspiração quântica tem sido propostos e aplicados com sucesso em problemas de otimização combinatória e numérica, apresentando desempenho superior àquele dos algoritmos evolutivos convencionais, quanto à melhoria da qualidade das soluções e à redução do número de avaliações necessárias para alcançá-las. Até o presente momento, no entanto, este novo paradigma de inspiração quântica ainda não havia sido aplicado à Programação Genética (PG), uma classe de algoritmos evolutivos que visa à síntese automática de programas de computador. Esta tese propõe, desenvolve e testa um novo modelo de algoritmo evolutivo com inspiração quântica, denominado Programação Genética Linear com Inspiração Quântica (PGLIQ), para a evolução de programas em código de máquina. A Programação Genética Linear é assim denominada porque cada um dos seus indivíduos é representado por uma lista de instruções (estruturas lineares), as quais são executadas sequencialmente. As contribuições deste trabalho são o estudo e a formulação inédita do uso do paradigma da inspiração quântica na síntese evolutiva de programas de computador. Uma das motivações para a opção pela evolução de programas em código de máquina é que esta é a abordagem de PG que, por oferecer a maior velocidade de execução, viabiliza experimentos em larga escala. O modelo proposto é inspirado em sistemas quânticos multiníveis e utiliza o qudit como unidade básica de informação quântica, o qual representa a superposição dos estados de um sistema deste tipo. O funcionamento do modelo se baseia em indivíduos quânticos, que representam a superposição de todos os programas do espaço de busca, cuja observação gera indivíduos clássicos e os programas (soluções). Nos testes são utilizados problemas de regressão simbólica e de classificação binária para se avaliar o desempenho da PGLIQ e compará-lo com o do modelo AIMGP (Automatic Induction of Machine Code by Genetic Programming), considerado atualmente o modelo de PG mais eficiente na evolução de código de máquina, conforme citado em inúmeras referências bibliográficas na área. 
Os resultados mostram que a Programação Genética Linear com Inspiração Quântica (PGLIQ) apresenta desempenho geral superior nestas classes de problemas, ao encontrar melhores soluções (menores erros) a partir de um número menor de avaliações, com a vantagem adicional de utilizar um número menor de parâmetros e operadores que o modelo de referência. Nos testes comparativos, o modelo mostra desempenho médio superior ao do modelo de referência para todos os estudos de caso, obtendo erros de 3 a $31 \%$ menores nos problemas de regressão simbólica, e de 36 a 39\% nos problemas de classificação binária. Esta pesquisa conclui que o paradigma da inspiração quântica pode ser uma abordagem competitiva para se evoluir programas eficientemente, encorajando o aprimoramento e a extensão do modelo aqui apresentado, assim como a criação de outros modelos de programação genética com inspiração quântica.

\section{Palavras-chave}

Aprendizado de máquina; inteligência computacional; computação evolutiva; programação genética; algoritmos evolutivos com inspiração quântica. 


\section{Abstract}

Dias, Douglas Mota; Pacheco, Marco Aurélio Cavalcanti (Advisor). Quantum-Inspired Linear Genetic Programming. Rio de Janeiro, 2010. 97p. Doctoral Thesis - Departamento de Engenharia Elétrica, Pontifícia Universidade Católica do Rio de Janeiro.

The superior performance of quantum algorithms in some specific problems lies in the direct use of quantum mechanics phenomena to perform operations with data on quantum computers. This feature has originated a new approach, named Quantum-Inspired Computing, whose goal is to create classic algorithms (running on classical computers) that take advantage of quantum mechanics principles to improve their performance. In this sense, some quantum-inspired evolutionary algorithms have been proposed and successfully applied in combinatorial and numerical optimization problems, presenting a superior performance to that of conventional evolutionary algorithms, by improving the quality of solutions and reducing the number of evaluations needed to achieve them. To date, however, this new paradigm of quantum inspiration had not yet been applied to Genetic Programming (GP), a class of evolutionary algorithms that aims the automatic synthesis of computer programs. This thesis proposes, develops and tests a novel model of quantum-inspired evolutionary algorithm named Quantum-Inspired Linear Genetic Programming (QILGP) for the evolution of machine code programs. Linear Genetic Programming is so named because each of its individuals is represented by a list of instructions (linear structures), which are sequentially executed. The contributions of this work are the study and formulation of the novel use of quantum inspiration paradigm on evolutionary synthesis of computer programs. One of the motivations for choosing by the evolution of machine code programs is because this is the GP approach that, by offering the highest speed of execution, makes feasible large-scale experiments. The proposed model is inspired on multi-level quantum systems and uses the qudit as the basic unit of quantum information, which represents the superposition of states of such a system. The model's operation is based on quantum individuals, which represent a superposition of all programs of the search space, whose observation leads to classical individuals and programs (solutions). The tests use symbolic regression and binary classification problems to evaluate the performance of QILGP and compare it with the AIMGP model (Automatic Induction of Machine Code by Genetic Programming), which is currently considered the most efficient GP model to evolve machine code, as cited in numerous references in this field. The results show that Quantum-Inspired Linear Genetic Programming (QILGP) presents superior overall performance in these classes of problems, by achieving better solutions (smallest error) from a smaller number of evaluations, with the additional advantage of using a smaller number of parameters and operators that the reference 
model. In comparative tests, the model shows average performance higher than that of the reference model for all case studies, achieving errors 3-31\% lower in the problems of symbolic regression, and 36-39\% in the binary classification problems. This research concludes that the quantum inspiration paradigm can be a competitive approach to efficiently evolve programs, encouraging the improvement and extension of the model presented here, as well as the creation of other models of quantum-inspired genetic programming.

\section{Keywords}

Machine learning; computational intelligence; evolutionary computation; genetic programming; quantum-inspired evolutionary algorithms. 


\section{Sumário}

1 Introdução $\quad \mathbf{1 5}$

$\begin{array}{ll}1.1 \text { Motivação } & 15\end{array}$

$\begin{array}{lll}1.2 \text { Objetivos } & 17\end{array}$

$\begin{array}{lll}1.3 & \text { Contribuições } & 18\end{array}$

1.4 Descrição do Trabalho 19

1.5 Organização do Trabalho 20

2 Fundamentos $\quad 21$

2.1 Mecânica Quântica 21

2.2 Computação Quântica 25

2.3 Algoritmos com Inspiração Quântica 27

2.4 Programação Genética 31

2.5 Programação Genética Linear $\quad 35$

3 Programação Genética Linear com Inspiração Quântica 40

3.1 Plataforma 40

3.2 Representação 41

3.3 Avaliação de um Indivíduo Clássico 46

3.4 Operador Quântico 48

3.5 Estrutura e Funcionamento do Modelo 49

3.6 Algoritmo Evolutivo $\quad 51$

4 Experimentos $\quad \mathbf{5 8}$

4.1 Configuração dos Modelos $\quad 61$

4.2 Estudos de Caso 64

5 Conclusões e Trabalhos Futuros $\quad \mathbf{8 0}$

5.1 Desempenho 81

5.2 Subpopulações $\quad 82$

$\begin{array}{lll}5.3 & \text { Iniciação dos Indivíduos } & 82\end{array}$

5.4 Código Não-Efetivo e Crescimento dos Programas 83

5.5 Aceleração por Processadores Gráficos $\quad 84$

A Código dos Melhores Programas Evoluídos 94 


\section{Lista de figuras}

2.1 Representação gráfica das probabilidades de se observar os valores 0 e 1 para um qubit qualquer.

2.2 Pseudocódigo do algoritmo evolutivo com inspiração quântica e representação binária QEA.

2.3 Representação típica de PG linear para programas.

2.4 Exemplo de um possível formato de uma instrução em PG linear.

2.5 Cruzamento de dois pontos entre dois indivíduos em PG Linear. 38

3.1 Cromossomo de um indivíduo clássico.

3.2 Exemplo da implementação de um qudit.

3.3 Criação de um indivíduo clássico (IC) pela observação de um indivíduo quântico (IQ).

3.4 Criação de um gene pela observação de um gene quântico. 46

3.5 Trecho de código relativo à avaliação de um caso de aptidão. 47

3.6 Pseudocódigo da implementação do operador $P$.

3.7 Curvas das probabilidades de um quadrit durante aplicações sucessivas do operador $P$.

3.8 Diagrama descritivo básico do modelo PGLIQ.

3.9 Pseudocódigo do algoritmo evolutivo da PGLIQ.

3.10 Pseudocódigo relativo à aplicação do operador $P$ em um indivíduo quântico.

4.1 Gráfico evolutivo do estudo de caso "Distância Euclideana" em função do tempo médio de cada execução dos modelos.

4.2 Gráfico evolutivo do estudo de caso "Distância Euclideana" em função do número médio de indivíduos avaliados a cada execução dos modelos.

4.3 Superfície representando a função "Chapéu Mexicano".

4.4 Gráfico evolutivo do estudo de caso "Chapéu Mexicano" em função do tempo médio por execução.

4.5 Gráfico evolutivo do estudo de caso "Chapéu Mexicano" em função do número médio de indivíduos avaliados por execução. 68

4.6 Gráfico evolutivo do estudo de caso "Composição Química" em função do tempo médio de cada execução dos modelos.

4.7 Gráfico evolutivo do estudo de caso "Composição Química" em função do número médio de indivíduos avaliados a cada execução dos modelos.

4.8 Gráfico evolutivo do estudo de caso "Pontos Quânticos” em função do tempo médio de cada execução dos modelos.

4.9 Gráfico evolutivo do estudo de caso "Pontos Quânticos" em função do número médio de indivíduos avaliados a cada execução dos modelos.

4.10 Representação gráfica do problema "Duas Espirais". 
4.11 Gráfico evolutivo do estudo de caso "Duas Espirais" em função do tempo médio de cada execução dos modelos.

4.12 Gráfico evolutivo do estudo de caso "Duas Espirais" em função do número médio de indivíduos avaliados a cada execução dos modelos.

4.13 Gráfico evolutivo do estudo de caso "Diagnóstico Cardíaco" em função do tempo médio de cada execução dos modelos.

4.14 Gráfico evolutivo do estudo de caso "Diagnóstico Cardíaco" em função do número médio de indivíduos avaliados a cada execução dos modelos.

A.1 Melhor programa evoluído pelo modelo AIMGP para o estudo de caso "Distância Euclideana".

A.2 Melhor programa evoluído pelo modelo PGLIQ para o estudo de caso "Distância Euclideana".

A.3 Melhor programa evoluído pelo modelo AIMGP para o estudo de caso "Chapéu Mexicano".

A.4 Melhor programa evoluído pelo modelo PGLIQ para o estudo de caso "Chapéu Mexicano". 


\section{Lista de tabelas}

2.1 Probabilidades de observação de cada um dos possíveis estados do indivíduo quântico.

3.1 Descrição das instruções.

41

3.2 Exemplo de um conjunto de funções e seus tokens. 42

4.1 Configuração do modelo AIMGP para os experimentos. 61

4.2 Configuração do modelo PGLIQ para os experimentos. 63

4.3 Comparação de média e desvio padrão das aptidões dos melhores indivíduos por geração para o estudo de caso "Distância Euclideana".

4.4 Comparação do número de acertos obtidos no estudo de caso "Distância Euclideana".

4.5 Comparação de média e desvio padrão das aptidões dos melhores indivíduos por geração para o estudo de caso "Chapéu Mexicano".

4.6 Comparação de média e desvio padrão das aptidões dos meIhores indivíduos por geração para o estudo de caso "Chapéu Mexicano" (ambos os modelos executados com demes).

4.7 Comparação de média e desvio padrão das aptidões dos melhores indivíduos por geração para o estudo de caso "Composição Química".

4.8 Comparação da aptidão dos melhores indivíduos obtidos pelos modelos para o estudo de caso "Composição Química". 72

4.9 Comparação de média e desvio padrão das aptidões dos melhores indivíduos por geração para o estudo de caso "Pontos Quânticos".

4.10 Comparação de média e desvio padrão das aptidões dos melhores indivíduos por geração para o estudo de caso "Duas Espirais".

4.11 Comparação da aptidão dos melhores indivíduos obtidos pelos modelos para o estudo de caso "Duas Espirais".

4.12 Comparação de média e desvio padrão das aptidões dos melhores indivíduos por geração para o estudo de caso "Diagnóstico Cardíaco".

4.13 Comparação da aptidão dos melhores indivíduos obtidos pelos modelos para o estudo de caso "Diagnóstico Cardíaco". 


\title{
Lista de acrônimos
}

\author{
AE Algoritmo Evolutivo \\ AEIQ Algoritmo Evolutivo com Inspiração Quântica \\ AG Algoritmo Genético
}

AIMGP Automatic Induction of Machine Code by Genetic Programming

CE Computação Evolutiva

CISC Complex Instruction Set Computing

CPU Central Processing Unit

EC Erro de Classificação

FPU Floating-Point Unit

GPGPU General Purpose Computing on Graphics Processing Unit

GPU Graphics Processing Unit

gsm Gerações Sem Melhoria

IC Indivíduo Clássico

IQ Indivíduo Quântico

MAE Mean Absolute Error

MAPE Mean Absolute Percentage Error

PC Personal Computer

PG Programação Genética

PGLIQ Programação Genética Linear com Inspiração Quântica

QEA Quantum-Inspired Evolutionary Algorithm

QF Qudit de Função

QILGP Quantum-Inspired Linear Genetic Programming

QT Qudit de Terminal

RISC Reduced Instruction Set Computing

SIMD Single Instruction, Multiple Data

SPARC Scalable Processor Architecture

TA Taxa de Acerto

TF Token de Função

TT Token de Terminal

XML Extensible Markup Language 